\title{
Evaluation of pH-sensitive poly(2-hydroxyethyl methacrylate-co-2-(diisopropylamino)ethyl methacrylate) copolymers as drug delivery systems for potential applications in ophthalmic therapies/ocular delivery of drugs
}

\author{
P. A. Faccia ${ }^{1}$, F. M. Pardini ${ }^{2}$, J. I. Amalvy $y^{1,2,3,4^{*}}$ \\ ${ }^{1}$ Instituto de Investigaciones Fisicoquímicas Teóricas y Aplicadas (INIFTA), (CCT La Plata CONICET- UNLP), Diag. \\ 113 y 64. La Plata, Argentina \\ ${ }^{2}$ Centro de Investigación y Desarrollo en Tecnología de Pinturas (CIDEPINT, CIC-CCT La Plata CONICET), Av. 52 \\ entre 121 y 122; La Plata, Argentina \\ ${ }^{3}$ Cátedra de Materiales Poliméricos de la Facultad de Ingeniería, Universidad Nacional de La Plata, Calle 1 y 47. La Plata, \\ Argentina \\ ${ }^{4}$ CITEMA. Facultad Regional La Plata (Universidad Tecnológica Nacional) 60 y 124, La Plata, Argentina
}

Received 29 October 2014; accepted in revised form 1 January 2015

\begin{abstract}
Smart polymers like $\mathrm{pH}$ sensitive systems can improve different pharmacological treatment. In this work the behavior of copolymers containing 2-hydroxyethyl methacrylate (HEMA) with different proportions of 2-(diisopropylamino)ethyl methacrylate (DPA) and different amounts of cross-linker agent, ethylene glycol dimethacrylate (EGDMA) are evaluated as $\mathrm{pH}$-sensitive drug delivery systems for potential application in ophthalmic therapies. A detailed characterization of the $\mathrm{pH}$-responsive behavior was performed by swelling studies and scanning electron microscopy (SEM) analysis. Drug loading and release studies at different $\mathrm{pH}$ values were evaluated using Rhodamine 6G (Rh6G) as a model drug. The interaction between Rh6G and hydrogels was studied by Fourier Transform Infrared (FTIR) spectroscopy and scanning electron microscopy (SEM). The results show that the presence of DPA in the copolymers confers $\mathrm{pH}$-responsive properties to the polymer, as noted in swelling and SEM studies, when the $\mathrm{pH}$ decreases below 7.40 the swelling degree increases and a porous morphology is observed. The apparent $p K_{\mathrm{a}}$ of copolymers was estimated between 6.80 and 7.17 depending on the composition. The amount of Rh6G loaded depends mainly on the medium $\mathrm{pH}$ and the interaction between the drug and the copolymers, observed by SEM and FTIR spectrum. The release of Rh6G of copolymers p(HEMA/DPA) show a normal Fickian or anomalous diffusion behavior at different $\mathrm{pH}$ values, depending on the HEMA/DPA ratio.
\end{abstract}

Keywords: smart polymers, 2-hydroxyethyl methacrylate, 2-(diisoproylamino)ethyl methacrylate, pH-sensitive hydrogels, drug delivery systems

\section{Introduction}

Hydrogels formed by chemical or physical crosslinking are three dimensional structures made from hydrophilic polymers that can imbibe a considerable amount of water while maintaining their integrity. The study of hydrogels as drug delivery systems and biomedical devices has been extensive over the last few decades because of their biocompatibility properties and control of solute transport [1-3]. More recently, sensitive hydrogels prepared with additional functions have gained considerable attention. In this way, the incorporation of stimuli-sensitive monomers in the chain of the hydrogel can improve the performance of these materials by increasing

\footnotetext{
${ }^{*}$ Corresponding author, e-mail: jamalvy@inifta.unlp.edu.ar (C) BME-PT
} 
responsiveness in a particular medium [4]. These hydrogels, often called 'intelligent' or 'smart' hydrogels, can undergo relatively large and abrupt, physical or chemical modification in response to changes in environmental conditions such as $\mathrm{pH}$, ionic strength, temperature or in presence of specific chemical compounds $[5,6]$. For this reason, they are usually known as environmentally sensitive hydrogels. These types of stimuli-responsive polymers have the property to swell, shrink, bend, or degrade in response to changes in the environmental conditions.

Due to its properties, sensitive hydrogels have been proposed for a number of applications like drug delivery, separation techniques and sensors [7-9]. Recently sensitive hydrogels have been also proposed for ocular drug delivery systems in order to improve the ocular bioavailability of drugs, and to reduce the appearance of side effects [10]. In this case, within topical application of drugs, the presence of ocular compact barrier in the corneal and conjunctival epithelia of the eye, along with the dynamics of the lacrimal system, hinder the drug absorption into the intraocular area $[11,12]$. The use of sensitive polymeric hydrogel allows to extend the residence time in the eye and to increase the percentage of drug absorbed [13].

In recent years research was mainly focusing to the technological innovations in this field with the aim to design hydrogels for a specific use as ocular drug delivery systems or in order to improve the uptake capacity and the release performance of these systems $[8,11,14]$ although few works have been published using intelligent systems.

Of particular interest in this work it is the use of smart polymers as ocular drug delivery systems, and specifically the use of $\mathrm{pH}$-sensitive hydrogels. This kind of material has been extensively studied as drug delivery systems for different applications, mainly due to the fact that they can release the drug selectively according to the $\mathrm{pH}$ of the medium [15]. In the human body there are variations in the physiologic $\mathrm{pH}$ values in both normal and pathological conditions, for example the gastrointestinal tract presents heterogeneous environments with different $\mathrm{pH}$ values ranging from 1 to 7.5 [16]. These conditions allow $\mathrm{pH}$-sensitive hydrogels to release the desired drug in the right place [17]. In case of ophthalmologic therapies the release should be close to the medium ocular $\mathrm{pH}$ of $7.45[18,19]$.
The potential use of stimuli-sensitive hydrogels allow not only a spatial control but also a temporal control; i.e. during the period of time when the $\mathrm{pH}$ value is outside the normal range. These $\mathrm{pH}$-sensitive hydrogels as drug delivery systems are potentially useful in ophthalmic therapies due to the fact that deviations from normal $\mathrm{pH}$ were observed in some disease processes, for example in ocular rosacea (an inflammation of the eye) [20]. Ocular pH change also in allergy and other conditions such as dry eye and bacterial infections [21]. Therefore these systems may be useful for controlling drug release in response to the pathological conditions.

Moreover, $\mathrm{pH}$-sensitive hydrogels are normally prepared by adding pendant acidic or basic functional groups to the polymer backbone by including during the polymerization monomers like N-(3aminopropyl)methacrylamide, 2-(dimethylamino) ethyl methacrylate, methacrylic acid and 2-aminoethyl methacrylate and 2-hidroxyethyl methacrylate (HEMA) for ionic type and butyl methacrylate, allyl diglycol carbonate, diallyl phthalate, and methyl methacrylate as neutral hydrophobic types [22-27]. Recently, we have reported the synthesis of a new $\mathrm{pH}$-sensitive hydrogel based on 2-(diisopropylamino) ethyl methacrylate (DPA) and 2-hidroxyethyl methacrylate (HEMA), p(HEMA-co-DPA) [28] with good film properties depending on the monomer ratio.

In this contribution, we evaluate the properties of these $\mathrm{pH}$-sensitive hydrogels with different proportions of HEMA and DPA and two degrees of crosslinking as potential materials for using in ocular drug delivery systems. Owing to this material, physical or chemical modifications can undergo in response to changes in environmental conditions, it is necessary to characterize the morphology and swelling behavior in function of the $\mathrm{pH}$ to understand and predict the drug's release rate. In this work we studied the swelling behavior of hydrogels in a range of $\mathrm{pH}$ from 5.5 to 8.4 which is the used range in eye drop solutions, and at the average ocular temperature of $34.5 \pm 0.5^{\circ} \mathrm{C}[29,30]$. We have determined $p K_{\mathrm{a}}$ values of different copolymers' compositions, and also we have studied morphological changes on hydrated samples at different medium $\mathrm{pH}$ values using Scanning Electron Microscopy (SEM). Their potential for ophthalmological application as $\mathrm{pH}$-sensitive control drug release system was investigated in vitro using Rhodamine $6 \mathrm{G}$ Chloride $\left(\mathrm{Rh} 6 \mathrm{G}, M_{\mathrm{w}}=\right.$ 479.01) as a model drug, because it is stable in 
aqueous solutions (water solubility at $25^{\circ} \mathrm{C}=$ $20 \mathrm{mg} \cdot \mathrm{L}^{-1}$ ) and it is easily detected by its UV absorption. Effects of HEMA/DPA contents and different cross-linking degrees on drug uptake and release behavior were studied at different $\mathrm{pH}$ values under ocular conditions. Despite the number of recent studies incorporating active agent into hydrogels [31], the interaction between the matrix and the drug receive limited attention in the literature and it is often overlooked [4]. That is why in this work we intended to analyze also hydrogels' performance and their interactions with the model drug by using FTIR and SEM analysis.

\section{Experimental section}

\subsection{Materials}

2-Hydroxyethyl methacrylate (HEMA, 97\%) and the cross-linker, ethylene glycol dimethacrylate (EGDMA, 98\%), were purchased from SigmaAldrich, USA, while 2-(diisopropylamino)ethyl methacrylate (DPA) were purchased from Scientific Polymers Products, USA. Darocur TPO (97\%) from Sigma-Aldrich, USA, was used as the initiator. The phosphate buffer solutions (PBS) were prepared from standard chemicals. The study of the hydrogels as drug delivery systems was performing using Rhodamine 6G Chloride (Rh6G, 95\%), from SigmaAldrich, USA, as model drug (Figure 1). This molecule is frequently used for those studies because it has a similar chemical structure compared to drugs $[32,33]$. Additionally Rh6G have good solubility in water $\left(20 \mathrm{~g} / \mathrm{L}\right.$ at $\left.25^{\circ} \mathrm{C}\right)$, chemical stability and it is easy detectable with UV-visible spectroscopy.

\subsection{Polymer synthesis}

The synthesis of DPA homopolymer and copolymers was performed in bulk by free radical poly-

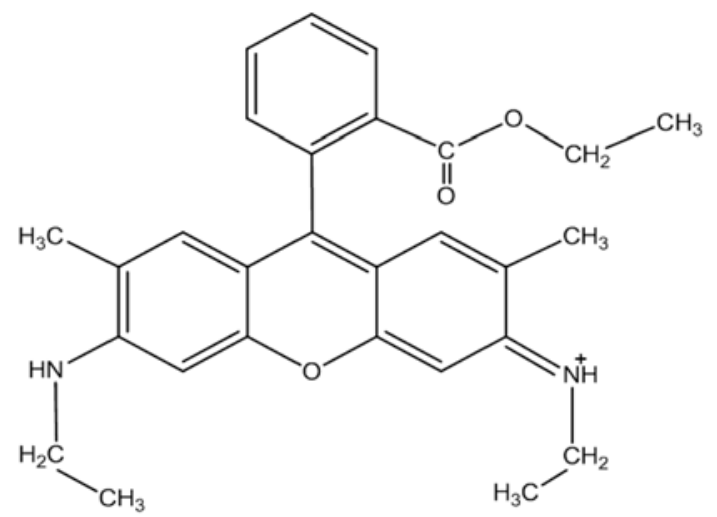

Figure 1. Chemical structure of Rhodamine $6 \mathrm{G}$ merization using diphenyl (2,4,6-trimethylbenzoyl)phosphine oxide (Darocur ${ }^{\circledR}$ TPO) as photo-initiator. Different ratios of HEMA/DPA monomers (namely $100 / 0,90 / 10$ and $70 / 30$ ) and 1 and $3 \mathrm{wt} \%$ of crosslinker (relative to the whole monomer) were mixed with $1 \% \mathrm{w} / \mathrm{v}$ of photo-initiator and irradiated with an UV-lamp (Rayonet RPR3500, USA). Films with $180 \pm 30 \mu \mathrm{m}$ of thickness were cut into circular pieces of $13 \mathrm{~mm}$ of diameter with a cork borer and dried at $25.0^{\circ} \mathrm{C}$ for $48 \mathrm{~h}$ before use. Film samples were denoted by using a short-hand notation HDX/Y-n, where $\mathrm{X}$ and $\mathrm{Y}$ denote the HEMA (H) and DPA (D) ratio respectively, and $\mathrm{n}$ denote the amount of crosslinker. More details of synthesis and experimental procedures can be found in a previous paper [28]. The experimental composition ratios in copolymers HD90/10-1, HD90/10-3, HD70/30-1 and HD70/30-3 are $82 / 18,85 / 15,66 / 34$ and $65 / 35$, respectively and they were determined by IR spectroscopy following the reported method by Canto and Pessan [34].

\subsection{Swelling degree}

For the determination of the swelling degree, dry samples were immersed in phosphate buffer solutions (PBS, $0.1 \mathrm{M}$ ) at the desired $\mathrm{pH}$ (ranging from 5.5 to 8.4 ) at the average ocular temperature of $34.5^{\circ} \mathrm{C}$ [30]. At regular periods of time samples were removed from the aqueous solution, blotted with filter paper to remove surface liquid, weighed and returned to the same container until reaching a constant weight. The equilibrium swelling degree $\left(Q_{\mathrm{e}}\right)$ was calculated using Equation (1):

$Q_{\mathrm{e}}[\%]=\frac{W_{\mathrm{e}}-W_{\mathrm{d}}}{W_{\mathrm{d}}} \cdot 100$

where $W_{\mathrm{d}}$ is the weight of the dry film and $W_{\mathrm{e}}$ is the weight of swollen film at equilibrium. Experiments were performed in triplicate.

\subsection{Drug loading}

Films were loaded with Rh6G by soaking the dry films into $20 \mathrm{~mL}$ of the drug solution $(50 \mathrm{mg} / \mathrm{L})$ in PBS at $\mathrm{pH} 6.5$ and 8.4 , and at $25.0^{\circ} \mathrm{C}$, until the equilibrium was reached (see below). For practical purposes the loading time was unified in 7 days for all samples. Drug uptake kinetics were followed by measuring the absorbance of the solution by UVvisible spectroscopy at $348 \mathrm{~nm}$ using a Fluorat ${ }^{\mathbb{}}-02$ Panorama spectrophotometer, Lumex, Russia. Samples loaded with Rh6G were denoted by adding the 
letter $\mathrm{R}$ in brackets to the name of the sample (e.g. HD90/10-1(R)).

The partition coefficient of the drug through the hydrogel was calculated using the modified method of Sato and Kim [35, 36], as shown by Equation (2):

$K_{\mathrm{d}}=\frac{V_{\mathrm{s}}\left(C_{0}-C_{\mathrm{e}}\right)}{V_{\mathrm{m}} C_{\mathrm{e}}}$

where $V_{\mathrm{s}}$ is the volume of solution, $V_{\mathrm{m}}$ is the volume of polymer film, $C_{0}$ is the initial solute concentration and $C_{\mathrm{e}}$ is the solute concentration in the solution at the equilibrium time.

\subsection{Scanning electron microscopy (SEM)}

Hydrogels' morphology and drug distribution were observed by Scanning Electron Microscopy (SEM) with an FEI - Quanta 200 (The Netherlands) instrument, in high vacuum mode and operated at 15 or $20 \mathrm{kV}$ acceleration voltage. $\mathrm{p}$ (HEMA-co-DPA) and pHEMA hydrogels were equilibrated during $24 \mathrm{~h}$ in different buffer solutions and then were frozen at $-40^{\circ} \mathrm{C}$ in an alcoholic solution followed lyophilization under vacuum for $24 \mathrm{~h}$. In order to prevent sample-charging effects during the observation, fractured pieces of samples were mounted onto the surface of an aluminum SEM specimen holder and sputter-coated with a thin overlayer of gold before observation. Films loaded with Rh6G were prepared in the same way.

\subsection{Infrared spectroscopy (FTIR)}

FTIR spectra were measured in transmission mode using a FTIR Nicolet 380 spectrometer, Thermo Scientific, USA. Samples were loaded with Rh6G until the equilibrium was reached and then powdered and mixed with $\mathrm{KBr}$; disks were formed by pressing. FTIR spectra were obtained by recording 64 scans between 4000 and $400 \mathrm{~cm}^{-1}$ with a resolution of $4 \mathrm{~cm}^{-1}$. Spectra processing was performed using the software EZ Omnic.

\subsection{Drug release experiments}

Release experiments of Rh6G loaded p(HEMA-coDPA) films were conducted in media of different $\mathrm{pH}$ values. Drug loaded films were removed from the loading solution, wiped with filter paper to remove surface liquid and placed directly into the release solution. Drug release experiments were performed by immersing p(HEMA-co-DPA) films into $20 \mathrm{~mL}$ of PBS $(0.1 \mathrm{M})$ at $34.5^{\circ} \mathrm{C}$. The dynamic drug concen- tration in the PBS solution was monitored by measuring the absorbance at $526 \mathrm{~nm}$. The Rh6G concentration released as a function of time $(t)$ was adjusted to a power-law type relationship $[37,38]$ using the equation of Ritger-Peppas (Equation (3)):

$\frac{M_{\mathrm{t}}}{M_{\mathrm{e}}}=k t^{\mathrm{n}}$

Here $M_{\mathrm{t}}$ and $M_{\mathrm{e}}$ are the cumulative amount of drug released after a time $t$ and at infinite time, respectively, while $k$ is a constant related to kinetic behavior and experimental conditions and $n$ is the exponent depending on the release process. Data were fitted only up to $60 \%$ of drug release in order to apply Equation (3).

Parameters $k$ and $n$ were calculated from the intercept and the slope of Equation (4):

$\ln \frac{M_{\mathrm{t}}}{M_{\mathrm{e}}}=\ln k+n \ln t$

For Fickian diffusion processes, Equation (5) applies to calculate the diffusion coefficient ( $D_{\text {ip }}$ ), where $L$ is the thickness of the film:

$\frac{M_{\mathrm{t}}}{M_{\mathrm{e}}}=4\left(\frac{D_{\mathrm{ip}} t}{\pi L^{2}}\right)^{1 / 2}$

\section{Results and discussion}

\subsection{Swelling studies}

In $\mathrm{pH}$-sensitive systems the release rate of the drug is regulated by several factors as swelling degree, drug-matrix interaction, water content and the initial PA concentration $[39,40]$. However the swelling behavior as a function of $\mathrm{pH}$ has a principal role in drug release regulation, which makes this technique to be an important tool to predict the drug's rate release. In this section swelling results of hydrogels in a range of $\mathrm{pH}$ from 5.5 to 8.4 and the determination of $p K_{\mathrm{a}}$ values corresponding to the different compositions of the copolymers are presented. Figure 2 shows the equilibrium swelling degree for different HEMA/DPA ratios with 1 and $3 \mathrm{wt} \%$ of cross-linker at different $\mathrm{pH}$ values.

Hydrogels of pHEMA show a slight increase in the swelling degree when increasing the $\mathrm{pH}$ from 5.5 to 8.4. However, the difference of swelling degree over the $\mathrm{pH}$ range studied in this work is rather low. Similar results were observed by Brannon-Peppas and Peppas [41]. On the other hand, hydrogels of p(HEMA-co-DPA) show a significant increase of 


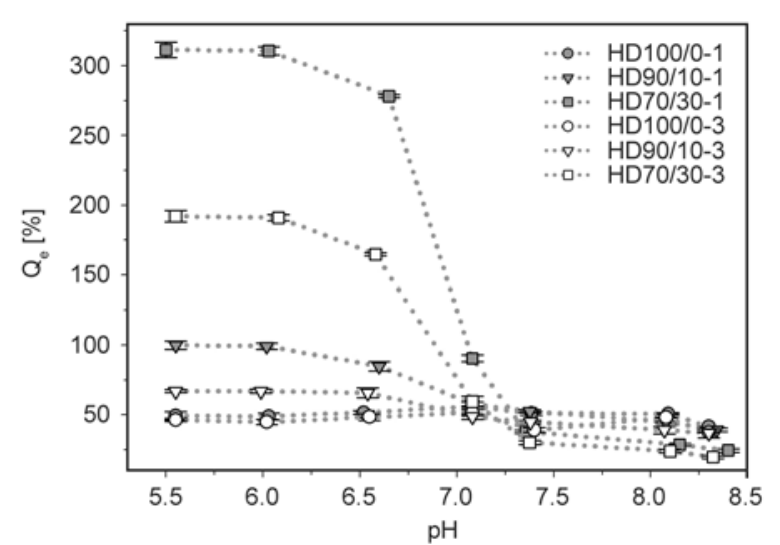

Figure 2. Equilibrium swelling degree for copolymers with different HEMA/DPA ratios, with 1 and $3 \mathrm{wt} \%$ of cross-linker as a function of $\mathrm{pH}$ at $34.5^{\circ} \mathrm{C}$

the swelling when the $\mathrm{pH}$ decrease below 7.40. This effect is directly proportional to the amount of DPA co-monomer present in copolymers, and is mainly attributed to the protonation of the tertiary amino groups. At $\mathrm{pH}$ below 7.40, amino groups become protonated and the electrostatic repulsion, between these ionized groups, expands the network space and increases its internal volume, allowing water to get into the matrix [14, 42, 43]. The equilibrium of swelling is reached around $\mathrm{pH}$ 6.0. At basic $\mathrm{pH}$, above 7.40, the effect is reversed, the swelling degree decreases with the amount of DPA present in the hydrogel. In this case functional groups of HEMA and DPA are able to form hydrogen-bonds [28], which in turn generate a proximity between polymer chains and consequently reduce the free space available for water molecules. Additionally, swelling values decrease also due to the hydrophobic nature of the unprotonated DPA moiety at basic $\mathrm{pH}$.

For all HEMA/DPA ratios the cross-linking density does not modify the sensitivity to respond to $\mathrm{pH}$ changes but it affects the swelling degree. For high proportion of cross-linking, the swelling decreases for a given $\mathrm{pH}$. This behavior is due mainly to two factors: first a matrix with higher crosslink density has less free space to be occupied by water; and second the crosslink degree generates a more rigid tridimensional structure which limits the chains' mobility and increases the elastic force that opposes to the expansion of the hydrogel's internal space [44]. The apparent copolymers' $p K_{\mathrm{a}}$ can be estimated by using swelling experiments at different $\mathrm{pHs}$. In the $\mathrm{pH}$ range of 6.5 to 7.4 the swelling of the hydrogel decreases almost linear when increasing $\mathrm{pH}$. In this range of $\mathrm{pH}$, both the protonated and unprotonated form of the DPA moiety are present inside the polymer matrix acting as a buffer system in the hydrogel. Under these conditions, the Handeson-Hasselbalch equation (Equation (6)) can be applied to determine the apparent $p K_{\mathrm{a}}$ :

$$
\begin{aligned}
& \mathrm{pH}=p K_{\mathrm{a}}+ \\
& +\log \left[\frac{\text { unprotonated state of tertiary amine group }}{\text { protonated state of tertiary amine group }}\right]
\end{aligned}
$$

The apparent $p K_{\mathrm{a}}$ of the hydrogel buffer system can be determined from the $\mathrm{pH}$ value for which the fraction between these two ionic forms is one. This corresponds to the point located in the middle of the swelling curve presented in Figure 2. The apparent $p K_{\mathrm{a}}$ values are show in Table 1 and range from 6.80 to 7.17 depending on polymers' composition and their crosslinking degree due to the availability of hydrogel's ionic groups to act as buffer system [29].

\subsection{Drug uptake}

Figure 3 shows the cumulative uptake of Rh $6 \mathrm{G}$ as a function of immersion time for pHEMA and $\mathrm{p}$ (HEMA-co-DPA) films in PBS at pH 6.5 (Figure $3 a$ ) and 8.4 (Figure $3 b$ ).

At $\mathrm{pH} 6.5$ by increasing the DPA content, the Rh6G kinetic uptake increases (Figure 3a). At this $\mathrm{pH}$ the swelling degree of hydrogels containing DPA (see above) favors the incoming of the drug into the film due to the increase of the network space and the diffusion of the aqueous solution, allowing the water soluble drug to get into the matrix. However, the final uptake and the partition coefficient at acid $\mathrm{pH}$ for pure pHEMA films are higher than that for the copolymer films (Table 2). This behavior can be explained in terms of an increased electrostatic repulsive interaction between the protonated tertiary amine groups of the polymer matrix and the positive charge on the Rh6G cation when increasing DPA content (see Figure 1 for the Rh6G chemical structure).

Table 1. Apparent $p K_{\mathrm{a}}$ values for copolymers at $34.5^{\circ} \mathrm{C}$

\begin{tabular}{|l|c|c|}
\hline \multicolumn{1}{|c|}{ Copolymers } & $\mathbf{p K}_{\mathbf{a}}$ & SD \\
\hline HD70/30-1 & 7.01 & 0.06 \\
\hline HD70/30-3 & 6.94 & 0.07 \\
\hline HD90/10-1 & 6.87 & 0.08 \\
\hline HD90/10-3 & 6.80 & 0.06 \\
\hline
\end{tabular}





Figure 3. Cumulative uptake of Rh6G as a function of immersion time for pHEMA and p(HEMA-co-DPA) films in PBS at $\mathrm{pH} 6.5$ (a) and 8.4 (b)

Table 2. Final mass uptake and partition coefficient $\left(K_{\mathrm{d}}\right)$ of $\mathrm{Rh} 6 \mathrm{G}$ at $\mathrm{pH} 6.5$ and 8.4

\begin{tabular}{|c|c|c|c|c|c|c|c|}
\hline \multirow{3}{*}{ HEMA/DPA } & \multirow{3}{*}{$\begin{array}{c}\text { Crosslinking } \\
\text { [wt\%] }\end{array}$} & \multicolumn{6}{|c|}{ Rh6G uptake (mg/g-dryfilm) } \\
\hline & & \multicolumn{3}{|c|}{ pH 6.5} & \multicolumn{3}{|c|}{ pH 8.4} \\
\hline & & Mean & SD & $\mathbf{K}_{\mathbf{d}}$ & Mean & SD & $\mathbf{K}_{\mathrm{d}}$ \\
\hline \multirow{2}{*}{$100 / 0$} & 1 & 27.4 & 0.1 & 902 & 31.8 & 0.3 & 1210 \\
\hline & 3 & 24.6 & 2.0 & 761 & 31.4 & 0.9 & 1066 \\
\hline \multirow{2}{*}{$90 / 10$} & 1 & 6.3 & 0.6 & 101 & 23.7 & 0.8 & 694 \\
\hline & 3 & 5.2 & 1.6 & 76 & 20.0 & 0.2 & 509 \\
\hline \multirow{2}{*}{$70 / 30$} & 1 & 4.7 & 1.1 & 26 & 18.0 & 0.9 & 491 \\
\hline & 3 & 3.7 & 0.9 & 28 & 15.1 & 0.2 & 358 \\
\hline
\end{tabular}

SD: standard deviation; n: number of measure between 2-4.

On the opposite, at $\mathrm{pH} 8.4$, by increasing the DPA content, the Rh6G kinetic uptake decreases (Figure $3 \mathrm{~b}$ ), and it is slower than at acid $\mathrm{pH}$. The equilibrium time ranges from 150 minutes for HD70/30 to 6 days for HD100/0, when loading is at $\mathrm{pH} 6.5$; and 2 days for HD70/30 and 6 days for HD100/0, when loading is at $\mathrm{pH} 8.4$.

At $\mathrm{pH} 8.4$ the increment on the DPA content causes a decrease in the swelling degree and consequently a decrease in the network space that retard the drug incorporation. However, the final uptake of $\mathrm{Rh} 6 \mathrm{G}$ at $\mathrm{pH} 8.4$ is higher than at $\mathrm{pH} 6.5$. At acidic $\mathrm{pH}$, the swelling increases due to the protonation of the functional group of the DPA, but the incorporation of $\mathrm{Rh} 6 \mathrm{G}$ decreased by the electrostatic repulsion between the tertiary amine of DPA (partially protonated) and the cation of Rhodamine 6G. At basic $\mathrm{pH}$, the electrostatic repulsion is less pronounced due to the decrease in the degree of ionization of the matrix, and hence the incorporation of Rh6G in the copolymers is higher. In conclusion, the amount of Rh6G incorporated into the polymer is inversely proportional with the swelling of the hydrogel, and depends mainly on the medium $\mathrm{pH}$ and the interaction between the drug and the copolymers' matrix.

At both $\mathrm{pH}$ values the total uptake Rh6G is higher for pure pHEMA homopolymer than for the copolymers. By including DPA monomer, the total amount of $\mathrm{OH}$ groups present in the hydrogel is reduced (as verified by FTIR) and, consequently, the available interaction sites decrease and then the number of Rh6G molecules incorporated also decreases (see Table 2).

As expected from the swelling data, increasing the degree of cross-linking from 1 to $3 \mathrm{wt} \%$ reduces the amount of Rh6G incorporated in all cases. Thus it is possible to modify the final incorporation of Rh6G changing the $\mathrm{pH}$ of the load medium instead of modifying the loading time. This allows regulating the amount of loaded drug into the hydrogel depending on the dose that is to be released.

\subsection{SEM characterization}

SEM is probably the best method for characterizing the hydrogel structure, especially in drug delivery systems because it offers information of surface 

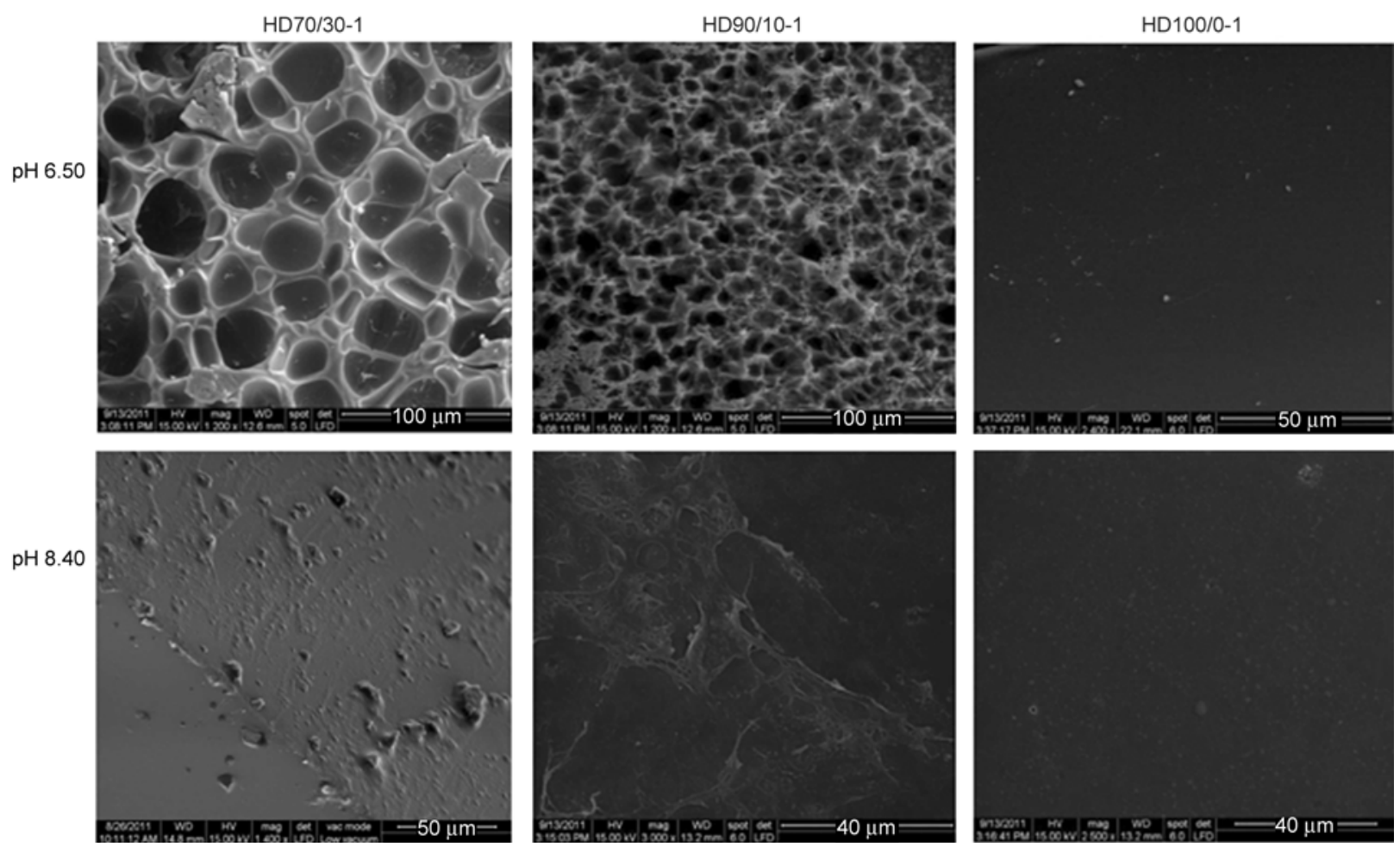

Figure 4. SEM images of hydrogel HD70/30-1, HD90/10-1 and HD100/0-1 at pH 6.5 and pH 8.4

porosity, amorphous and crystalline characterization, particle size, phase separation and in particular the active principle ingredient distribution in the structure [45]. Morphologic changes of lyophilized $\mathrm{pH}-$-responsive hydrogels, after exposure them to aqueous solutions of different $\mathrm{pH}$ values ( 6.5 and 8.4), have been examined by SEM technique. Images are shown in Figure 4.

The surface of the hydrogel HD70/30-1 at $\mathrm{pH} 6.5$ shows an open morphology state with a porous structure, thin walls and a predominant free space as a consequence of the matrix expansion at this $\mathrm{pH}$. At $\mathrm{pH}$ to 8.40 a collapsed state is observed with almost a featureless structure due to lower swelling degree and a more hydrophobic polymer at this $\mathrm{pH}$. For hydrogel HD90/10-1 the surface also shows a morphologic change with the $\mathrm{pH}$ value. At $\mathrm{pH} 6.50$ a homo-



a)

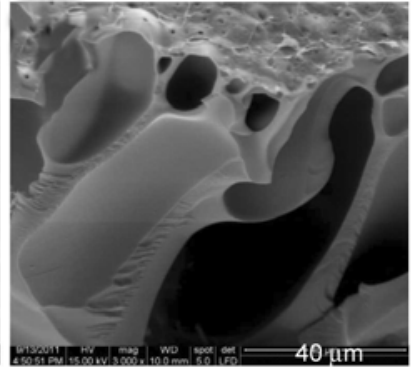

b) geneous pore distribution on the surface is observed, while at $\mathrm{pH} 8.40$ a non-porous and compact surface is appreciated. When the DPA content is higher, the equilibrium swelling increases, which led to more and larger pores in hydrogels as obtained from lyophilization, being $5 \pm 2 \mu \mathrm{m}$ for $10 \mathrm{wt} \%$ and $7 \pm 2 \mu \mathrm{m}$ for the $30 \%$ of DPA. By comparing those values with the mesh sizes of conventional hydrogels, smaller than $100 \mathrm{~nm}[46,47]$, both systems have a higher pore size. For hydrogel HD100/0-1 no changes with $\mathrm{pH}$ are appreciated and in all cases a compact surface is observed. The incorporation of DPA confers pH-responsive properties to the polymer, as noted in swelling studies; therefore changing the medium $\mathrm{pH}$ not only changes the film volume but also the morphology. In the case of sample with higher concentration of cross-linker ( $3 \mathrm{wt} \%$ ) the same trend in

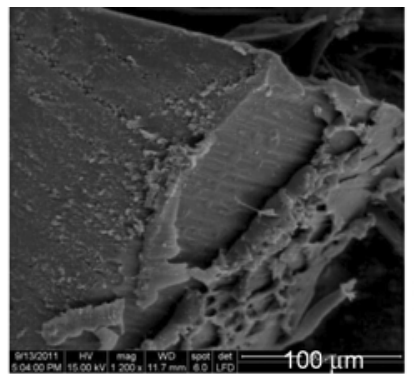

c)

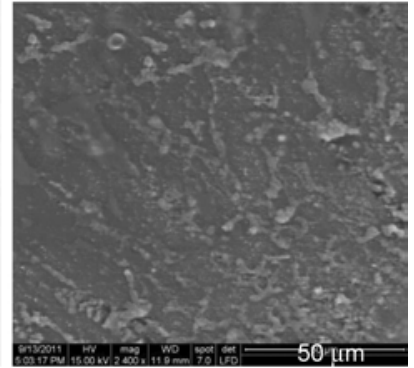

d)

Figure 5. SEM images of hydrogel HD70/30-1(R) loaded at $\mathrm{pH}$ 6.5: a) surface, b) inside the matrix; and at pH 8.4: c) inside the matrix, d) surface 
morphological changes with the medium $\mathrm{pH}$ is observed (data not shown).

In Figure 5 are presented SEM images of samples HD70/30-1(R), loaded with RhG6 at pH 6.5 and 8.4 .

SEM images of the hydrogel 70/30-1(R) loaded at both pHs (6.5 and 8.4) show the presence of Rh6G. In the case of loading at $\mathrm{pH} 6.5$, the surface (Figure 5a) shows a greater accumulation of Rh6G unlike inside the matrix (Figure 5b). While at $\mathrm{pH}$ 8.4 the Rh6G is observed both inside the matrix and on the surface (Figure $5 \mathrm{c}$ and $5 \mathrm{~d}$ respectively). Rh6G molecules have lower affinity for the matrix of the copolymer HD70/30-1 at acidic $\mathrm{pH}$, and therefore incorporation is lower and mainly superficial.

\subsection{FTIR spectroscopy}

Figure 6 shows the FTIR spectra of HD70/30-1 and HD100/0-1 with (samples labeled R) and without Rh6G. The main differences in the high wavenumbers region of the spectrum are the increasing intensity of the stretching band of the $\mathrm{O}-\mathrm{H}$ group $\left(3414 \mathrm{~cm}^{-1}\right)$. The existence of an interaction between Rh6 G and- $\mathrm{OH}$ groups through the group $=\mathrm{N}+(\mathrm{H})$ is known, therefore interactions between Rh6G and pHEMA [48] are also expected. The $\mathrm{C}-\mathrm{H}$ stretching region is also different. Upon the copolymerization with DPA, the $-\mathrm{CH}_{2}-$ and $-\mathrm{CH}_{3}$ bands of the stretching modes of pure pHEMA observed at 2986, 2951 and $2882 \mathrm{~cm}^{-1}$ (Figure 6) are overlapped with those of the DPA monomer and peaks become broader. In the $\mathrm{C}-\mathrm{H}$ stretching region of the HD70/30-1 copolymer spectrum, a broad band centered at $2965 \mathrm{~cm}^{-1}$ is observed. These wavenumbers correspond to the characteristic peak of the methine group in the isopropyl moiety $\left(\left(\mathrm{CH}_{3}\right)_{2}-\mathrm{CH}-\right)$ [49]. However after Rh6G loading the peak pattern of the FTIR changed showing peaks at 2985,2950 and $2888 \mathrm{~cm}^{-1}$. These

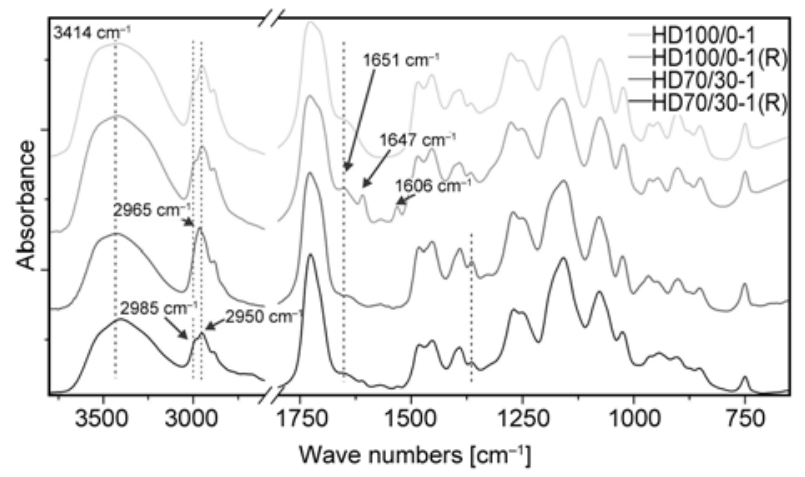

Figure 6. FTIR spectra for films: HD100/0-1, HD100/01(R), HD70/30-1 y HD70/30-1(R) peaks values are close to the $\mathrm{C}-\mathrm{H}$ stretching of the pure pHEMA, suggesting that the peak of the stretching of the methine group at $2965 \mathrm{~cm}^{-1}$ shifted, probably to higher wavenumbers and overlapping with the $2985 \mathrm{~cm}^{-1}$ peak due to the loss of interaction between the lone electron pair of tertiary amine group of DPA with the OH of pHEMA [28] and the formation of new hydrogen bonds with the Rh6G molecules [48].

In the $2000-400 \mathrm{~cm}^{-1}$ region the new features after Rh6G loading are mainly in low wavenumbers side of the $\mathrm{C}=\mathrm{O}$ (free) stretching band (located at $1730 \mathrm{~cm}^{-1}$ ), namely a band at $1651 \mathrm{~cm}^{-1}$ from the contribution of bonded carbonyl groups of Rh6G, and small peaks at 1647 and $1606 \mathrm{~cm}^{-1}$ from the xanthene ring of this molecule (see $[50,51]$ for more details of Rh6G spectrum). These contributions are more evident in HD100/0-1 film due to its higher loading of Rh6G. In HD70/30-1 film, the band of the isopropyl group $\left(\left(\mathrm{CH}_{3}\right)_{2}-\mathrm{CH}-\right)$ of DPA moiety, observed at $1336 \mathrm{~cm}^{-1}$ in the unloading film, shows lower intensity after Rh6G loading. Minor differences are also observed in the 1000$880 \mathrm{~cm}^{-1}$ region, where the contribution of the absorption bands of $\mathrm{Rh} 6 \mathrm{G}$ is negligible. This region is associated to $\mathrm{C}-\mathrm{C}$ modes of the carbon backbone of the polymer.

In summary, observed differences in the FTIR spectra between loaded and unloaded films indicate that Rh6G molecules are interacting with polymer chains, probably with both parts of the copolymer (HEMA and DPA moieties) by hydrogen bonding or through dipole-dipole interaction [52].

\subsection{Drug release}

\subsubsection{Effect of medium $\mathrm{pH}$}

Figure 7 shows the cumulative concentration of $\mathrm{Rh} 6 \mathrm{G}$ released at $34.5^{\circ} \mathrm{C}$ in $\mathrm{PBS}$ for different $\mathrm{pH}$ values as a function of time for HD70/10-1, HD90/10-1 and HD100/0-1 loaded at pH 8.4. This $\mathrm{pH}$ was chosen because the drug uptake is the highest found in this work (see section 3.2 and Table 2.) In copolymers of HEMA/DPA release kinetics of $\mathrm{Rh} 6 \mathrm{G}$ varies significantly with changing the $\mathrm{pH}$ of the medium, as the $\mathrm{pH}$ increases the release becomes faster. This effect is related to the swelling property of these hydrogels when changing the medium $\mathrm{pH}$. At basic $\mathrm{pH}$ the matrix is closed and the swelling is low, by acidifying the medium, the hydrogel swells and the release rate of the Rh6G increases as well as 


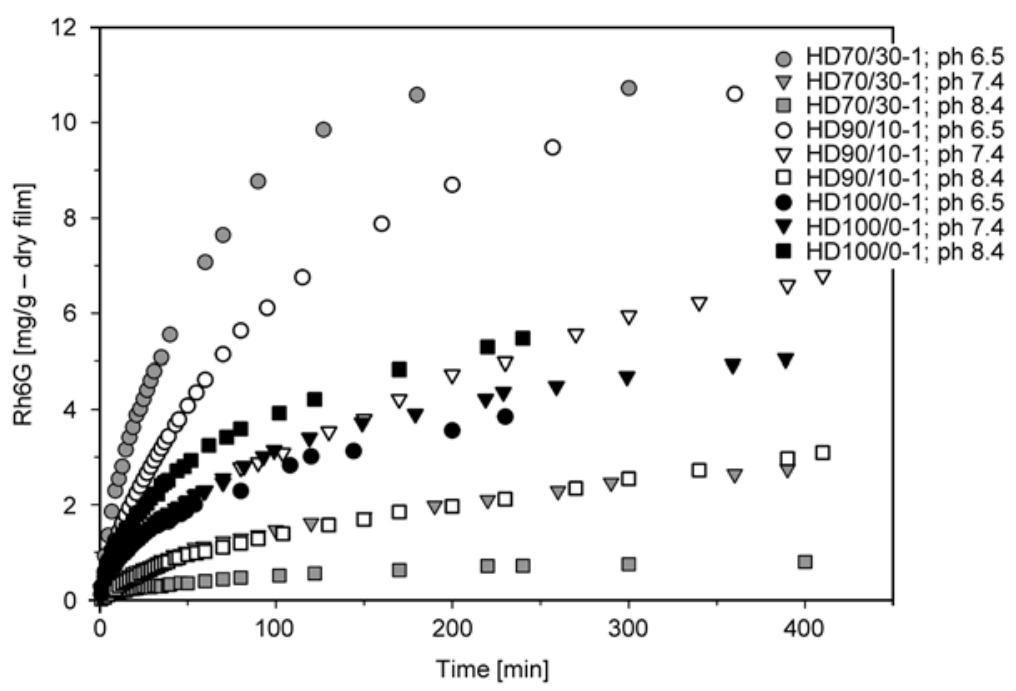

Figure 7. Cumulative concentration of Rh6G released as a function of time for HD70/10-1, HD90/10-1 and HD100/0-1 (loaded at $\mathrm{pH} 8.4$ ) at $34.5^{\circ} \mathrm{C}$ in $\mathrm{PBS}$ for different $\mathrm{pH}$ values

the pore size of the hydrogel [53]. In the case of HD70/30-1 the effect of the $\mathrm{pH}$ on the release kinetics is much more pronounced than in HD90/10-1 copolymer due to the higher amount of DPA. At acid $\mathrm{pH}$, the electrostatic repulsion between the tertiary amine groups of the DPA partially protonated and the Rh6G cation favors the released of this drug.

Drug release behavior at different $\mathrm{pH}$ values is also consistent with morphologic characteristics observed in SEM images (Figure 3), the higher the pore size of the hydrogel, the faster is the released of the drug.

The total amount of Rh6G released from pHEMA film is incomplete (about $30 \%$ is released) and it is almost $\mathrm{pH}$ independent as a consequence of the interaction of the Rh6G molecule with the polymer functional groups, as observed by FTIR spectroscopy. Similar results were obtained in other cases of interaction between the drug and the matrix [5456]. Although, for DPA containing polymers, the total Rh6G released at pH 6.5 (about 90\%) is higher than at $\mathrm{pH} 8.4$ (about 40\%). At acidic $\mathrm{pH}$, the tertiary amine groups are partially protonated and the electrostatic interaction with the Rh6G cation impels the release from the matrix. At pH 8.4, the electrostatic repulsion is no longer acting and, therefore, the driving force is reduced.

Table 3 shows the kinetics parameters ( $k$ and $n$ ) of the experimental data of Figure 7, calculated using Equation (4), and the diffusion coefficient $\left(D_{\text {ip }}\right)$, using Equation (5).

For pure pHEMA samples $\mathrm{n}$ values indicate a Fickian transport from $\mathrm{pH} 6.5$ to 8.4 . Highly soluble
Table 3. Parameters $(k, n)$ calculated from the fit of Equation (4), and diffusion coefficients $\left(D_{\text {ip }}\right)$ calculated from Equation (5) for the Rh6G release curves from Figure 7

\begin{tabular}{|c|c|c|c|c|c|}
\hline Samples & $\mathbf{p H}$ & $\mathbf{R}^{\mathbf{2}}$ & $\begin{array}{c}\mathbf{k} \cdot \mathbf{1 0} \mathbf{2}^{\mathbf{2}} \\
{\left[\mathbf{c m}^{-1} \mathbf{]}\right.}\end{array}$ & $\mathbf{n}$ & $\begin{array}{c}\mathbf{D}_{\mathbf{i p}} \cdot \mathbf{1 0}^{\mathbf{9}} \\
{\left[\mathbf{c m}^{\mathbf{2}} \cdot \mathbf{s e g}^{-\mathbf{1}} \mathbf{]}\right.}\end{array}$ \\
\hline \multirow{3}{*}{ HD100/0-1 } & 6.5 & 0.996 & 2.75 & 0.51 & 0.82 \\
\cline { 2 - 6 } & 7.4 & 0.986 & 2.73 & 0.51 & 0.94 \\
\cline { 2 - 6 } & 8.4 & 0.998 & 3.31 & 0.50 & 1.23 \\
\hline \multirow{3}{*}{ HD90/10-1 } & 6.5 & 0.995 & 2.10 & 0.67 & - \\
\cline { 2 - 6 } & 7.4 & 0.995 & 1.58 & 0.63 & - \\
\cline { 2 - 6 } & 8.4 & 0.993 & 1.42 & 0.61 & - \\
\hline \multirow{3}{*}{ HD70/30-1 } & 6.5 & 0.978 & 3.17 & 0.78 & - \\
\cline { 2 - 6 } & 7.4 & 0.993 & 1.57 & 0.56 & - \\
\cline { 2 - 6 } & 8.4 & 0.988 & 1.84 & 0.48 & - \\
\hline
\end{tabular}

drugs, like Rh6G, typically exhibit Fickian release from hydrogels, and the release profile is mainly dependent upon the solubility and diffusion kinetics of the drug. For the hydrogel HD90/10-1, the $n$ values are between 0.5 and 1 , indicating anomalous transport, and a domination of relaxation process over diffusion. In the case of HD70/30-1, $n$ values varies significantly with the $\mathrm{pH}$, at $\mathrm{pH} 6.5$ and 7.4 are between 0.5 and 1 while at $\mathrm{pH} 8.4$ is low than 0.5 . For higher pHs experimental values suggest a different mechanism transport, that is, the presence of another process besides passive diffusion. Above $p K_{\mathrm{a}}$ the deprotonation is accompanied by a deswelling of the hydrogel (see Figure 2).

The $D_{\text {ip }}$ value of $\mathrm{Rh} 6 \mathrm{G}$ in water at $25^{\circ} \mathrm{C}$ is $4.14 \cdot 10^{-6} \mathrm{~cm}^{2} / \mathrm{s}$ and as expected, all values found in this work for Fickian diffusion are lower than this one [57]. 
In principle the relation between the $\mathrm{pH}$ and the percentage of released R6G indicate that, while the release mechanism can be similar for different $\mathrm{pHs}$, the final amount of drug released depends on the medium's $\mathrm{pH}$ and on the material's swelling degree. This behavior demonstrates the ability of such copolymers to achieve a control of drug released as a function of the $\mathrm{pH}$ of the medium.

\subsubsection{Effect of cross-linking density}

To evaluate the effect of cross-linker concentration on the mechanism of the drug transport at $\mathrm{pH} 7.4$ and $34.5^{\circ} \mathrm{C}$, parameters $(n, k)$ of the power law model in Equation (3) are calculated (Table 4).

The release of Rh6G in pHEMA homopolymer at $\mathrm{pH} 7.4$ seems to follow a Fickian diffusion behavior as suggested by values of $n$. However, by incorporating DPA, $n$ values are close to 0.6 for all contents and cross-linking densities, suggesting a non-Fickian behavior.

The mechanism of Rh6G release does not seem to be affected by increasing the cross-linking density for the same copolymer composition, as judged by $\mathrm{n}$ values. However, a reduction in the $\mathrm{k}$ parameter is the most important effect of higher cross-linker concentration. The decrease in kinetic constant values reflects the decrease in the rate of drug release, which might be due to the dominance of chain entanglement and the decrease in the water content of polymers with different cross-linking densities. By increasing the cross-linking density, pores are smaller and less water is allowed to enter the matrix. Since Rh6G is a water soluble molecule, this factor has an important impact on drug rate. The larger pore in the $1 \mathrm{wt} \%$ cross-linked copolymers allows Rh6G diffusion with no or little resistance compared to smaller one. Pore size is significantly impacted by the extent of cross-linking and their increasing values result in lower released kinetic.

Table 4. Parameters $(k, n)$ calculated from the fit of Equation (4) for the Rh6G release from different crosslinking densities at $\mathrm{pH} 7.4$ and $34.5^{\circ} \mathrm{C}$

\begin{tabular}{|c|c|c|c|c|}
\hline Samples & $\begin{array}{c}\text { Cross-linking } \\
{[\mathbf{w t} \% \mathbf{l}}\end{array}$ & $\mathbf{R}^{\mathbf{2}}$ & $\begin{array}{c}\mathbf{k} \cdot \mathbf{1 0}^{\mathbf{3}} \\
{\left[\mathbf{m i n}^{-\mathbf{1}}\right]}\end{array}$ & $\mathbf{n}$ \\
\hline \multirow{2}{*}{ HD100/0 } & 1 & 0.990 & 27.28 & 0.51 \\
\cline { 2 - 5 } & 3 & 0.989 & 20.72 & 0.51 \\
\hline \multirow{2}{*}{ HD90/10 } & 1 & 0.997 & 15.82 & 0.61 \\
\cline { 2 - 5 } & 3 & 0.994 & 10.87 & 0.59 \\
\hline \multirow{2}{*}{ HD70/30 } & 1 & 0.996 & 15.16 & 0.57 \\
\cline { 2 - 5 } & 3 & 0.980 & 7.67 & 0.61 \\
\hline
\end{tabular}

This behavior could be an advantage in the case of treatments where a prolonged therapy is required.

\section{Conclusions}

The incorporation of DPA confers $\mathrm{pH}$-responsive properties to the polymer, copolymers show a significant increase of the swelling degree when the $\mathrm{pH}$ decrease below 7.40 and reach the equilibrium around $\mathrm{pH}$ 6.0. This effect is directly proportional to the amount of present DPA in copolymers and inversely proportional with the amount of crosslinker. The apparent $p K_{\mathrm{a}}$ of copolymers depend on the composition of HEMA/DPA and the crosslinking degree of hydrogels, and estimated values are between 6.80 and 7.17. SEM images of copolymers show important morphological changes when varying the medium $\mathrm{pH}$ according to swelling results. At acid $\mathrm{pH}$ SEM imagines show an open morphology state with a porous structure as a consequence of the matrix expansion at this $\mathrm{pH}$, while at basic $\mathrm{pH}$ show a collapsed state due to lower swelling degree and a more hydrophobic polymer. Hydrogels with $30 \mathrm{wt} \%$ of DPA show higher pores than hydrogels with $10 \mathrm{wt} \%$. For hydrogel HD100/0-1 no changes with $\mathrm{pH}$ are appreciated and in all cases a compact surface is observed.

Copolymers of HEMA and DPA, having good film forming and physicochemical properties, were tested as drug delivery systems using Rh6G as model drug. The amount of Rh6G incorporated is higher for pure pHEMA than for copolymers and depends mainly on the medium $\mathrm{pH}$ and the interaction between the drug and the copolymers' matrix. The comparison of FTIR spectra between loaded and unloaded films indicates that $\mathrm{Rh} 6 \mathrm{G}$ molecules interact with the $\mathrm{OH}$ group of the HEMA by hydrogen bonding or through dipole-dipole interaction. At $\mathrm{pH} 6.5$, the total $\mathrm{Rh} 6 \mathrm{G}$

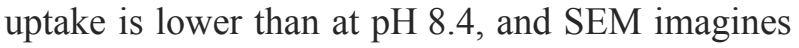
show a greater accumulation on the surface at this $\mathrm{pH}$. Thus the loaded Rh6G is inversely proportional with the swelling of the hydrogel and mainly depends on the interaction between the drug and the matrix of the copolymers. The total release of the drug depends on the polymer composition and medium $\mathrm{pH}$. For pure pHEMA, the drug remains strongly associated with the polymer chains inside the matrix and, therefore, its release is very slow. On the other hand, for copolymers, the total Rh6G released at acid $\mathrm{pH}$ is higher than at basic $\mathrm{pH}$, and it increases as the proportion of DPA monomer increases. The 
pore size observed from SEM images is highly correlated with the drug release behavior when varying the medium $\mathrm{pH}$. For copolymers, the release of the Rh6G model drug in PBS follows a non-Fickian diffusion process for $\mathrm{pHs}$ values less than or equal to 7.4. The change of the polymer's cross-linking density affects only the drug release rate.

In conclusion, by changing the DPA content and the degree of cross-linking density it is possible to modify kinetic parameters and, therefore, to control the release kinetics depending on the medium $\mathrm{pH}$. Results show that copolymers of HEMA/DPA are potentially useful as drug delivery systems for ophthalmic therapies.

\section{Acknowledgements}

We are grateful to CICPBA and ANPCyT (PICT 20110238) for their financial assistance. PAF is a postdoctoral student from CONICET (Argentina), FMP is Ph. D. student from CICPBA, and JIA is member of CICPBA. The authors thank Professor Piatti Guillermina for her assistance in reviewing and correcting the article.

\section{References}

[1] Jagur-Grodzinski J.: Polymeric gels and hydrogels for biomedical and pharmaceutical applications. Polymers for Advanced Technologies, 21, 27-47 (2010). DOI: $10.1002 /$ pat.1504

[2] Alvarez-Lorenzo C., Concheiro A.: Molecularly imprinted materials as advanced excipients for drug delivery systems. Biotechnology Annual Review, 12, 225-268 (2006).

DOI: $10.1016 / \mathrm{s} 1387-2656(06) 12007-4$

[3] Geever L. M., Cooney C. C., Lyons J. G., Kennedy J. E., Nugent M. J., Devery S., Higginbotham C. L.: Characterisation and controlled drug release from novel drug-loaded hydrogels. European Journal of Pharmaceutics and Biopharmaceutics, 69, 1147-1159 (2008). DOI: $10.1016 /$ j.ejpb.2007.12.021

[4] García D. M., Escobar J. L., Noa Y., Bada N., Hernáez E., Katime I.: Timolol maleate release from $\mathrm{pH}$-sensible poly(2-hydroxyethyl methacrylate- $c o$-methacrylic acid) hydrogels. European Polymer Journal, 40, 16831690 (2004).

DOI: $10.1016 /$ j.eurpolymj.2004.03.012

[5] Bettini R., Colombo P., Peppas N. A.: Solubility effects on drug transport through $\mathrm{pH}$-sensitive, swelling-controlled release systems: Transport of theophylline and metoclopramide monohydrochloride. Journal of Controlled Release, 37, 105-111 (1995).

DOI: $\underline{10.1016 / 0168-3659(95) 00069-K}$
[6] Atta A. M., Arndt K-F.: Swelling behavior of $\mathrm{pH}-$ and temperature-sensitive copolymers containing 2-hydroxyethyl methacrylate and $N$-vinyl-2-pyrrolidone crosslinked with new crosslinkers. Polymer International, 53, 1870-1881 (2004).

DOI: $10.1002 /$ pi.1606

[7] Peppas N. A., Khare A. R.: Preparation, structure and diffusional behavior of hydrogels in controlled release. Advanced Drug Delivery Reviews, 11, 1-35 (1993). DOI: 10.1016/0169-409X(93)90025-Y

[8] Xinming L., Yingde C., Lloyd A. W., Mikhalovsky S. V., Sandeman S. R., Howel C. A., Liewen L.: Polymeric hydrogels for novel contact lens-based ophthalmic drug delivery systems: A review. Contact Lens and Anterior Eye, 31, 57-64 (2008). DOI: 10.1016/j.clae.2007.09.002

[9] Richter A., Paschew G., Klatt S., Lienig J., Arndt K-F., Adler H-J.: Review on hydrogel-based $\mathrm{pH}$ sensors and microsensors. Sensors, 8, 561-581 (2008).

DOI: $10.3390 / \mathrm{s} 8010561$

[10] Casolaro M., Casolaro I., Lamponi S.: Stimuli-responsive hydrogels for controlled pilocarpine ocular delivery. European Journal of Pharmaceutics and Biopharmaceutics, 80, 553-561 (2012).

DOI: 10.1016/j.ejpb.2011.11.013

[11] Alvarez-Lorenzo C., Hiratani H., Concheiro A.: Contact lenses for drug delivery. American Journal of Drug Delivery, 4, 131-151 (2006). DOI: $10.2165 / 00137696-200604030-00002$

[12] Urtti A., Salminen L.: Minimizing systemic absorption of topically administered ophthalmic drugs. Survey of Ophthalmology, 37, 435-456 (1993). DOI: 10.1016/0039-6257(93)90141-S

[13] del Amo E. M., Urtti A.: Current and future ophthalmic drug delivery systems: A shift to the posterior segment. Drug Discovery Today, 13, 135-143 (2008). DOI: $10.1016 /$ j.drudis.2007.11.002

[14] Lin C-C., Metters A. T.: Hydrogels in controlled release formulations: Network design and mathematical modeling. Advanced Drug Delivery Reviews, 58, 1379-1408 (2006).

DOI: 10.1016/j.addr.2006.09.004

[15] Wang K., Xu X., Wang Y., Yan X., Guo G., Huang M., Luo F., Zhao X., Wei Y., Qian Z.: Synthesis and characterization of poly(methoxyl ethylene glycol-caprolactone-co-methacrylic acid-co-poly(ethylene glycol) methyl ether methacrylate) $\mathrm{pH}$-sensitive hydrogel for delivery of dexamethasone. International Journal of Pharmaceutics, 389, 130-138 (2010). DOI: $10.1016 /$ j.ijpharm.2010.01.026

[16] Evans D. F., Pye G., Bramley R., Clark A. G., Dyson T. J., Hardcastle J. D.: Measurement of gastrointestinal $\mathrm{pH}$ profiles in normal ambulant human subjects. Gut, 29, 1035-1041 (1988). DOI: $10.1136 /$ gut.29.8.1035 
[17] Wang Y., Chen L., Tan L., Zhao Q., Luo F., Wei Y., Qian Z.: PEG-PCL based micelle hydrogels as oral docetaxel delivery systems for breast cancer therapy. Biomaterials, 35, 6972-6985 (2014).

DOI: 10.1016/j.biomaterials.2014.04.099

[18] Carney L. G., Hill R. M.: Human tear pH. Diurnal variations. Archives of Ophthalmology, 94, 821-824 (1976).

DOI: 10.1001/archopht.1976.03910030405011

[19] Baeyens V., Gurny R.: Chemical and physical parameters of tears relevant for the design of ocular drug delivery formulations. Pharmaceutica Acta Helvetiae, 72, 191-202 (1997).

DOI: $10.1016 / \mathrm{S} 0031-6865(97) 00021-6$

[20] Jaros P. A., Coles W. H.: Ocular surface pH in rosacea. CLAO Journal, 9, 333-336 (1983).

[21] Norn M. S.: Tear fluid pH in normals, contact lens wearers, and pathological cases. Acta Ophthalmologica, 66, 485-489 (1988).

DOI: $10.1111 / j .1755-3768.1988 . t b 04368 . x$

[22] Bawa P., Pillay V., Choonara Y. E., du Toit L. C.: Stimuli-responsive polymers and their applications in drug delivery. Biomedical Materials, 4, 022001/1-022001/15 (2009).

DOI: $10.1088 / 1748-6041 / 4 / 2 / 022001$

[23] París R., Quijada-Garrido I.: Temperature- and pHresponsive behaviour of poly(2-(2-methoxyethoxy)ethyl methacrylate- $\mathrm{co}-\mathrm{N}, \mathrm{N}$-dimethylaminoethyl methacrylate) hydrogels. European Polymer Journal, 46, 21562163 (2010).

DOI: $10.1016 /$ j.eurpolymj.2010.09.004

[24] Emileh A., Vasheghani-Farahani E., Imani M.: Swelling behavior, mechanical properties and network parameters of $\mathrm{pH}$ - and temperature-sensitive hydrogels of poly((2-dimethyl amino) ethyl methacrylate-co-butyl methacrylate). European Polymer Journal, 43, 19861995 (2007).

DOI: 10.1016/j.eurpolymj.2007.02.002

[25] Andrade-Vivero P., Fernandez-Gabriel E., AlvarezLorenzo C., Concheiro A.: Improving the loading and release of NSAIDs from pHEMA hydrogels by copolymerization with functionalized monomers. Journal of Pharmaceutical Sciences, 96, 802-813 (2007).

DOI: 10.1002/jps.20761

[26] Amalvy J. I., Wanless E. J., Li Y., Michailidou V., Armes S. P., Duccini Y.: Synthesis and characterization of novel ph-responsive microgels based on tertiary amine methacrylates. Langmuir, 20, 8992-8999 (2004).

DOI: $10.1021 / 1 \mathrm{a} 049156 \mathrm{t}$

[27] Wang K., Fu S. Z., Gu Y. C., Xu X., Dong P. W., Guo G., Zhao X., Wei Y. Q., Qian Z. Y.: Synthesis and characterization of biodegradable $\mathrm{pH}$-sensitive hydrogels based on poly( $\varepsilon$-caprolactone), methacrylic acid, and poly(ethylene glycol). Polymer Degradation and Stability, 94, 730-737 (2009).

DOI: $10.1016 /$ j.polymdegradstab.2008.12.013
[28] Faccia P. A., Amalvy J. I.: Synthesis, characterization, and swelling behavior of new $\mathrm{pH}$-sensitive hydrogels derived from copolymers of 2-hydroxyethyl methacrylate and 2-(diisopropylamino)ethylmethacrylate. Journal of Applied Polymer Science, 127, 1974-1980 (2013). DOI: $10.1002 /$ app. 37576

[29] Firestone B. A., Dickason M. A., Tran T.: Solubility characteristics of three fluoroquinolone ophthalmic solutions in an in vitro tear model. International Journal of Pharmaceutics, 164, 119-128 (1998). DOI: $10.1016 / \mathrm{S} 0378-5173(97) 00427-4$

[30] Milder B.: The lacrimal apparatus, in 'Adler's Physiology of the eye: Clinical application' (eds.: Moses R. A.) Mosby, St. Louis, 15-35 (1987).

[31] Xu X., Fu S., Wang K., Jia W., Guo G., Zheng X., Dong P., Guo Q., Qian Z.: Preparation and characterization of vitamin-12 loaded biodegradable $\mathrm{pH}$-sensitive microgels. Journal of Microencapsulation, 26, 642648 (2009).

DOI: $\underline{10.3109 / 02652040802610827}$

[32] Desai S., Perkins J., Harrison B. S., Sankar J.: Understanding release kinetics of biopolymer drug delivery microcapsules for biomedical applications. Materials Science and Engineering: B, 168, 127-131 (2010). DOI: $10.1016 /$ j.mseb.2009.11.006

[33] Ozay O.: Synthesis and characterization of novel $\mathrm{pH}-$ responsive poly(2-hydroxylethyl methacrylate- $\mathrm{CO}-\mathrm{N}$ allylsuccinamic acid) hydrogels for drug delivery. Journal of Applied Polymer Science, 131, 39660/139660/10 (2014).

DOI: $10.1002 / a p p .39660$

[34] Canto L. B., Pessan L. A.: Determination of the composition of styrene-glycidyl methacrylate copolymers by FTIR and titration. Polymer Testing, 21, 35-38 (2002).

DOI: 10.1016/S0142-9418(01)00041-1

[35] Sato S., Kim S. W.: Macromolecular diffusion through polymer membranes. International Journal of Pharmaceutics, 22, 229-255 (1984). DOI: 10.1016/0378-5173(84)90024-3

[36] Varshosaz J., Koopaie N.: Cross-linked poly (vinyl alcohol) hydrogel: Study of swelling and drug release behaviour. Iranian Polymer Journal 11, 123-131 (2002).

[37] Ritger P. L., Peppas N. A.: A simple equation for description of solute release II. Fickian and anomalous release from swellable devices. Journal of Controlled Release, 5, 37-42 (1987).

DOI: 10.1016/0168-3659(87)90035-6

[38] Ritger P. L., Peppas N. A.: A simple equation for description of solute release I. Fickian and non-Fickian release from non-swellable devices in the form of slabs, spheres, cylinders or discs. Journal of Controlled Release, 5, 23-36 (1987).

DOI: 10.1016/0168-3659(87)90034-4

[39] Bajpai A. K., Shukla S. K., Bhanu S., Kankane S.: Responsive polymers in controlled drug delivery. Progress in Polymer Science, 33, 1088-1118 (2008). DOI: $10.1016 /$ j.progpolymsci.2008.07.005 
[40] Karlgard C. C. S., Wong N. S., Jones L. W., Moresoli C.: In vitro uptake and release studies of ocular pharmaceutical agents by silicon-containing and p-HEMA hydrogel contact lens materials. International Journal of Pharmaceutics, 257, 141-151 (2003).

DOI: 10.1016/S0378-5173(03)00124-8

[41] Brannon-Peppas L., Peppas N. A.: Equilibrium swelling behavior of $\mathrm{pH}$-sensitive hydrogels. Chemical Engineering Science, 46, 715-722 (1991).

DOI: 10.1016/0009-2509(91)80177-Z

[42] Chirila T. V., Chen Y-C., Griffin B. J., Constable I. J.: Hydrophilic sponges based on 2-hydroxyethyl methacrylate. I. Effect of monomer mixture composition on the pore size. Polymer International, 32, 221-232 (1993). DOI: 10.1002/pi.4990320303

[43] Nam K., Watanabe J., Ishihara K.: Modeling of swelling and drug release behavior of spontaneously forming hydrogels composed of phospholipid polymers. International Journal of Pharmaceutics, 275, 259-269 (2004). DOI: $10.1016 /$ j.ijpharm.2004.02.009

[44] Pinzón N., Espinosa A., Perilla J., Hernáez E., Katime I.: A model for swelling and solute diffusion in water soluble polymers (in Spanish). Revista Iberoamericana de Polímeros, 3, 38-54 (2002).

[45] Lowman A. M., Peppas N. A.: Encyclopedia of controlled drug delivery Vol 1. Wiley, New York (1999).

[46] Chen J., Park H., Park K.: Synthesis of superporous hydrogels: Hydrogels with fast swelling and superabsorbent properties. Journal of Biomedical Materials Research, 44, 53-62 (1999).

DOI: 10.1002/(SICI)1097-4636(199901)44:1<53::AIDJBM6>3.0.CO;2-W

[47] Chen J., Park K.: Superporous hydrogels: Fast responsive hydrogel systems. Journal of Macromolecular Science Part A: Pure and Applied Chemistry, 36, 917-930 (1999).

DOI: $10.1080 / 10601329908951189$

[48] Tleugabulova D., Sui J., Ayers P. W., Brennan J. D.: Evidence for rigid binding of Rhodamine $6 \mathrm{G}$ to silica surfaces in aqueous solution based on fluorescence anisotropy decay analysis. Journal of Physical Chemistry B, 109, 7850-7858 (2005).

DOI: $10.1021 / \mathrm{jp} 045569 \mathrm{r}$
[49] Jin L., Deng Y., Hu J., Wang C.: Preparation and characterization of core-shell polymer particles with protonizable shells prepared by oxyanionic polymerization. Journal of Polymer Science Part A: Polymer Chemistry, 42, 6081-6088 (2004).

DOI: $10.1002 /$ pola.20453

[50] Watanabe H., Hayazawa N., Inouye Y., Kawata S.: DFT vibrational calculations of Rhodamine $6 \mathrm{G}$ adsorbed on silver: Analysis of tip-enhanced Raman spectroscopy. Journal of Physical Chemistry B, 109, 50125020 (2005).

DOI: 10.1021/jp045771u

[51] Tripathi S. K., Monga A., Saini G. S. S.: Characterization of thermally evaporated thin films of Rhodamine 6G. Smart Materials and Structures, 18, 125012/1125012/7 (2009). DOI: $10.1088 / 0964-1726 / 18 / 12 / 125012$

[52] Saini G. S., Kaur S., Tripathi S. K., Mahajan C. G., Thanga H. H., Verma A. L.: Spectroscopic studies of Rhodamine 6G dispersed in polymethylcyanoacrylate. Spectrochimica Acta Part A: Molecular and Biomolecular Spectroscopy, 61, 653-658 (2005).

DOI: $10.1016 /$ j.saa.2004.05.022

[53] Tomić S. L., Mićić M. M., Filipović J. M., Suljovrujić E. H.: Swelling and drug release behavior of poly(2hydroxyethyl methacrylate/itaconic acid) copolymeric hydrogels obtained by gamma irradiation. Radiation Physics and Chemistry, 76, 801-810 (2007). DOI: 10.1016/j.radphyschem.2006.05.013

[54] Momose T., Ito N., Kanai A., Watanabe Y., Shibata M.: Adsorption of levocabastine eye drops by soft contact lenses and its effects in rabbit eyes. CLAO Journal, 23, 96-99 (1997).

[55] Lumbroso P., Nhamias M., Nhamias S., Tranche P.: A preliminary study of the adsorption and release of preservatives by contact lenses and collagen shields. CLAO Journal, 22, 61-63 (1996).

[56] Miranda M. N., García-Castiñeiras S.: Effects of pH and some common topical ophthalmic medications on the contact lens Permalens. CLAO Journal, 9, 43-48 (1983).

[57] Gendron P-O., Avaltroni F., Wilkinson K. J.: Diffusion coefficients of several Rhodamine derivatives as determined by pulsed field gradient-nuclear magnetic resonance and fluorescence correlation spectroscopy. Journal of Fluorescence 18, 1093-1101 (2008).

DOI: $10.1007 / \mathrm{s} 10895-008-0357-7$ 